\title{
Endemic characteristics of infantile visceral leishmaniasis in the People's Republic of China
}

\author{
Qing Fu' ${ }^{1,2,3}$, Shi-Zhu Li ${ }^{1,2,3}$, Wei-Ping Wu ${ }^{1,2,3}$, Yan-Yan Hou ${ }^{4}$, Song Zhang ${ }^{4}$, Yu Feng ${ }^{5}$, Li-Ping Zhang ${ }^{6}$ \\ and Lin-Hua Tang ${ }^{1,2,3^{*}}$
}

\begin{abstract}
Background: Visceral leishmaniasis (VL) was once a severe parasitic disease in China. Thanks to the great efforts of integrated control, VL was eliminated in most epidemic areas, except for certain western provinces (autonomous region) at the end of 1950s. From then on, VL gained less attention and has seemed to spread, especially in the last 15 years. Infants are the most important population threatened by VL. However, there have been few studies on the endemic characteristics of infantile VL in China.

Methods: Infantile VL cases were collected from the online National Infectious Diseases Reporting System (NIDRS). Statistical description and inference was used to reveal the endemic characteristics in gender, age group, time and regionalism. Spatial analysis was carried out to explore the high risk area for infantile VL in China.

Results: A total of 1093 infantile VL cases were reported from 2006 to 2012. There was no statistically significant difference in gender over time. The minimum, maximum and mean age of these cases was 1.1, 35.9 and 13.8 months, respectively. Among them $86.92 \%$ were under 2 years of age, and there was a statistically significant difference among age groups over time. An incidence peak appeared in 2008-2009, most cases were distributed in the months September to December, and there was a tail-raising effect in the coming two months of the next year. More than $98 \%$ of cases were reported in Xinjiang Uygur Autonomous Region, Gansu Province and Sichuan Province, accounting for $61.02 \%, 32.75 \%$ and $4.57 \%$, respectively. A total of 56 counties reported infantile VL cases, with the cumulative incidence ranging from 0.02 to $24.57 \%$. There were two main zones of high endemicity for infantile VL in China. The monthly incidence clearly coincides with the number of towns where infantile VL cases were reported. Three fatalities were reported during the study period, the case fatality rate was $2.75 \%$.
\end{abstract}

Conclusions: The endemic situation of infantile $V L$ is serious, and there are several active foci of infantile $V L$ prevalence in China. VL has emerged as a severe threat to infants of endemic regions in China.

Keywords: Endemic, Infantile, Visceral leishmaniasis, China

\section{Background}

Visceral leishmaniasis (VL) is a potentially fatal parasitic disease, which is caused by several species of intracellular protozoans belonging to the genus Leishmania [1-3]. Infected individuals may develop the most severe clinical syndrome, characterized by prolonged fever, splenomegaly, weight loss, hepatomegaly and pancytopenia, with more than $90 \%$ case-fatality in the absence of treatment [4]. VL

\footnotetext{
*Correspondence: ipdtlh@sh163.net

'National Institute of Parasitic Diseases, Chinese Center for Disease Control and Prevention, Shanghai 200025, People's Republic of China

${ }^{2}$ Key Laboratory of Parasite and Vector Biology, Ministry of Health, Shanghai 200025, People's Republic of China

Full list of author information is available at the end of the article
}

is endemic in more than 62 countries, with an estimated 500000 new patients each year worldwide [5,6]. In the past, VL severely prevailed in 16 of 34 provinces (municipalities or autonomous regions) of the People's Republic of China [7]. After the implementation of the comprehensive control campaign, VL had been eliminated in most eastern provinces of China $[8,9]$. However, in western provinces (autonomous regions), VL is still a serious health problem, the protozoan parasites are Leishmania donovani and Leishmania infantum, transmitted by the sandfly Phlebotomus longiductus, Phlebotomus wui and Phlebotomus chinensis [10,11]. Infants are the important population threatened by VL in China, new cases

\section{Biomed Central}


ceaselessly occur each year, which should be taken seriously. The objective of this article is to evaluate the endemic status on infantile VL in China, as well as to provide control suggestions.

\section{Methods}

Definition of infantile VL

A child who is infected by VL from the time of birth to approximately 3 years of age.

\section{Source of data}

Data were collected from the online National Infectious Diseases Reporting System (NIDRS) from January 1st 2006 to December 31st 2012, which covered all notifiable diseases, including VL. Each record included a unique reference number, name, gender, date of birth and diagnosis, home address, etc. The inaccurate and repeated case reporting records were excluded, only the final-judgment cases were saved. Relevant population data was collected from the departments of immunization of provincial Centers for Disease Control and Prevention (CDC) and the national census database from the Bureau of Statistics. The electronic boundaries of counties were retrieved from the national fundamental geographic information system.

\section{Quality control}

Provincial CDCs were responsible for checking and revising the local case reporting records. The National Institute of Parasitic Diseases will check and verify each case reported from the non-endemic areas. Furthermore, an assessment in 4 counties, namely Kashi City, Shufu County, Shule County and Jiashi County was conducted to evaluate the data quality in terms of the precision, reliability, completeness and concordance.

\section{Data analysis}

Infantile VL cases were grouped by gender, age group, time and regionalism, respectively, the Mathsoft Axum 7.0 and SPSS12.0 was used for epidemiological analysis. The cumulative incidence of infantile VL of each county was calculated and analyzed in ArcGIS 9.3 for spatial analysis of infantile VL. The level of statistical significance was set at $P=0.05$.

\section{Ethics statement}

The study protocol was approved by the Ethics Review Committee of the National Institute of Parasitic Disease of China CDC. Data were collected from the online NIDRS, we did not carry out any field investigation, detection and experiments on patients or their sample. Therefore, there were no ethical issues.

\section{Results}

Data quality

From January 1st 2010 to June 30th 2012, 196 VL paper reporting cards from 4 counties were compared with those of NIDRS. The underreporting rate was $2.55 \%$ (5/196), the repeat rate was $14.80 \%(29 / 196)$, completion rate was $83.67 \%(164 / 196)$, and the concordance rate of key items in NIDRS compared with those on the cards was $97.96 \%$ $(192 / 196)$.

\section{Endemic characteristics \\ Gender distribution}

During the period from January 1st 2006 to December 31st 2012, 1093 infantile VL cases were reported in NIDRS (Table 1). As shown, 59.56\% (651/1093) were male, and the sex ratio was $147: 100$. There was no statistically significant difference in gender over time $\left(\chi^{2}=8.60, P>0.05\right)$.

\section{Age distribution}

Infants were divided into three age groups, from birth to 1 year (not including 1 year), 1 to 2 years (not including 2 years) and 2 to 3 years (not including 3 years). During the study period, the number of cases of the three age groups was 507, 443 and 143, respectively. The minimum, maximum and mean age of the total cases was 1.1 months, 35.9 months and 13.8 months, respectively. As shown $86.92 \%$ (950/1093) of cases were infants under 2 years of age (Table 1$)$. There was a statistically significant difference among age groups over time $\left(\chi^{2}=82.50\right.$, $P<0.05)$, the number of VL cases in the 2 to 3 year age group was significantly less than that of the other two groups.

\section{Time distribution}

The annual average of 156 infantile VL cases was reported during the study period. It showed that there was an incidence peak in 2008-2009 (Figure 1), accounting for $52.88 \%$ (578/1093). Cases were grouped by

Table 1 Gender and age distribution of infantile VL cases in China

\begin{tabular}{lcccccc}
\hline \multirow{2}{*}{ Year } & \multicolumn{2}{c}{ Gender } & & \multicolumn{3}{c}{ Age* group } \\
\cline { 2 - 3 } \cline { 6 - 7 } & Male & Female & & $(\mathbf{0 - 1}) \mathbf{y}$ & {$[\mathbf{1 - 2}) \mathbf{y}$} & {$[\mathbf{2 - 3} \mathbf{y}$} \\
\hline 2006 & 46 & 17 & & 27 & 30 & 6 \\
2007 & 69 & 48 & & 56 & 54 & 7 \\
2008 & 168 & 128 & & 179 & 94 & 23 \\
2009 & 162 & 120 & & 138 & 106 & 38 \\
2010 & 98 & 68 & & 70 & 70 & 26 \\
2011 & 62 & 30 & & 18 & 50 & 24 \\
2012 & 46 & 31 & & 19 & 39 & 19 \\
Total & 651 & 442 & 507 & 443 & 143 \\
\hline
\end{tabular}

* Age $=$ date of diagnosis - date of birth. 


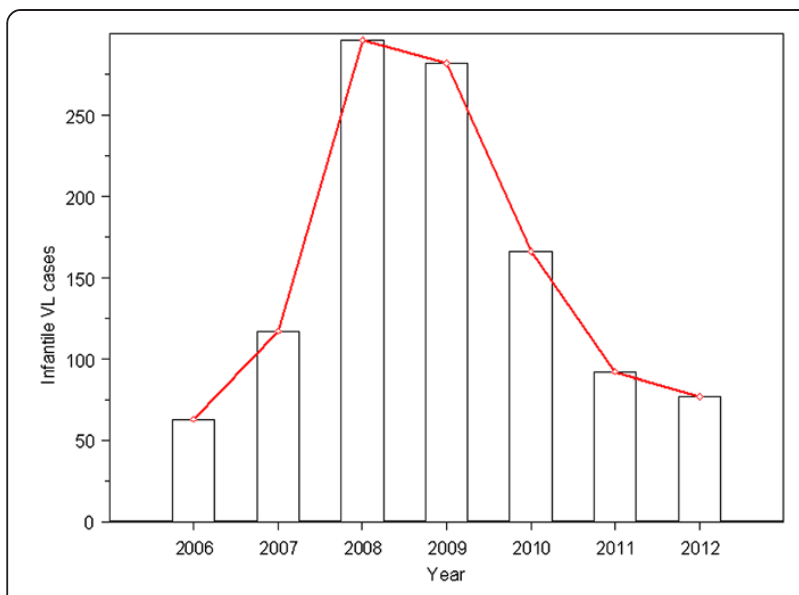

Figure 1 The yearly number of infantile VL cases in China from 2006 to 2012.

month, 53.07\% (580/1093) distributed in the months September to December, which were the peak time of incidence of infantile VL. The monthly number of infantile VL cases indicated a strongly seasonal distribution pattern, with a peak in autumn according to the Chinese lunar calendar. Moreover, there was a tailraising effect in the coming two months of the next year (Figure 2).

\section{Geographical distribution}

A total of 8 provinces (autonomous regions) reported infantile VL cases (Table 2). Most of the cases were distributed in Xinjiang Uygur Autonomous Region, Gansu Province and Sichuan Province, which accounted for $61.02 \%(667 / 1093), 32.75 \%(358 / 1093)$ and $4.57 \%$ (50/1093), respectively. Sporadic infantile VL cases were reported in Inner Mongolia Autonomous Region,

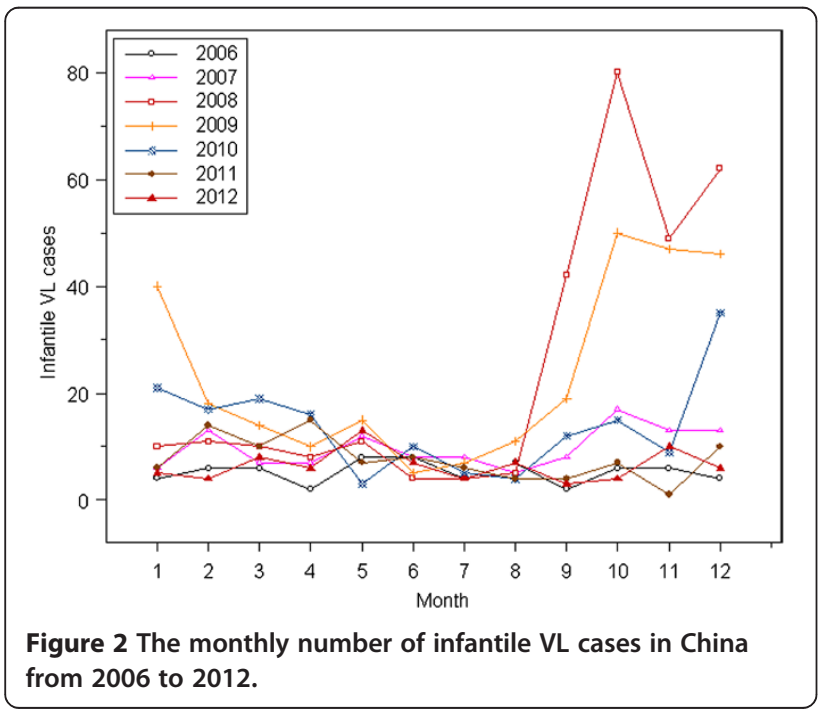

Shanxi Province and Shaanxi Province. Furthermore, 3 infantile VL cases were reported as imported cases, two of which were in Zhejiang Province, and the other one was in Hunan Province.

56 counties (cities) reported infantile VL cases during the study period. Grouped by county, the cumulative incidence of infantile VL of each county was calculated and joined to the map layer in ArcGIS 9.3, with county name matching. Cumulative incidences were divided into 3 quantities with gradual colors (Figure 3). Eleven counties with red color indicated the cumulative incidence was higher than $3.00 \%$, three of which were over $10.00 \%$, they are Wen County of Gansu Province (24.57\%), Jiashi County of Xinjiang Uygur Autonomous Region (17.31\%) and Zhouqu County of Gansu Province (11.04\%o). In addition, Wudu City and Diebu County of Gansu Province, Yuli County of Xinjiang Uygur Autonomous Region, and Heishui County of Sichuan Province, their cumulative incidence was all higher than $6.00 \%$. Cumulative incidences were analyzed in Spatial Analyst extension, and contours were created on the basis of density analysis, As a result, there were two main zones (IandII) of high endemicity for infantile VL in China from 2006 to 2012.

The number of infantile VL cases and the number of towns with infantile VL cases of each year were grouped by month, the relationship between the monthly number of cases and towns is presented in Figure 4. It showed that there was a large peak of infantile VL incidence that occurred in December 2008 (82 cases), and the monthly number of cases clearly coincides with the number of towns where infantile VL cases were reported.

\section{Deaths}

Of these 1093 well-documented infantile VL cases, three fatalities were reported in 2007 (Table 3), the case fatality rate was $2.75 \%$.

Table 2 Geographical distribution of infantile VL cases in China

\begin{tabular}{|c|c|c|c|c|c|c|c|c|}
\hline \multirow{2}{*}{$\begin{array}{c}\text { Province } \\
\text { (Autonomous } \\
\text { Region) } \\
\end{array}$} & \multicolumn{7}{|c|}{ Year } & \multirow[t]{2}{*}{ Total } \\
\hline & 2006 & 2007 & 2008 & 2009 & 2010 & 2011 & 2012 & \\
\hline Xinjiang & 23 & 40 & 249 & 205 & 105 & 18 & 27 & 667 \\
\hline Gansu & 33 & 64 & 39 & 67 & 55 & 60 & 40 & 358 \\
\hline Sichuan & 4 & 11 & 8 & 8 & 6 & 8 & 5 & 50 \\
\hline Inner Mongolia & 1 & 1 & 0 & 1 & 0 & 0 & 0 & 3 \\
\hline Shanxi & 1 & 0 & 0 & 1 & 0 & 3 & 2 & 7 \\
\hline Shaanxi & 0 & 1 & 0 & 0 & 0 & 1 & 3 & 5 \\
\hline Zhejiang* & 1 & 0 & 0 & 0 & 0 & 1 & 0 & 2 \\
\hline Hunan* & 0 & 0 & 0 & 0 & 0 & 1 & 0 & 1 \\
\hline
\end{tabular}

* Cases were confirmed as imported cases from other endemic province. 


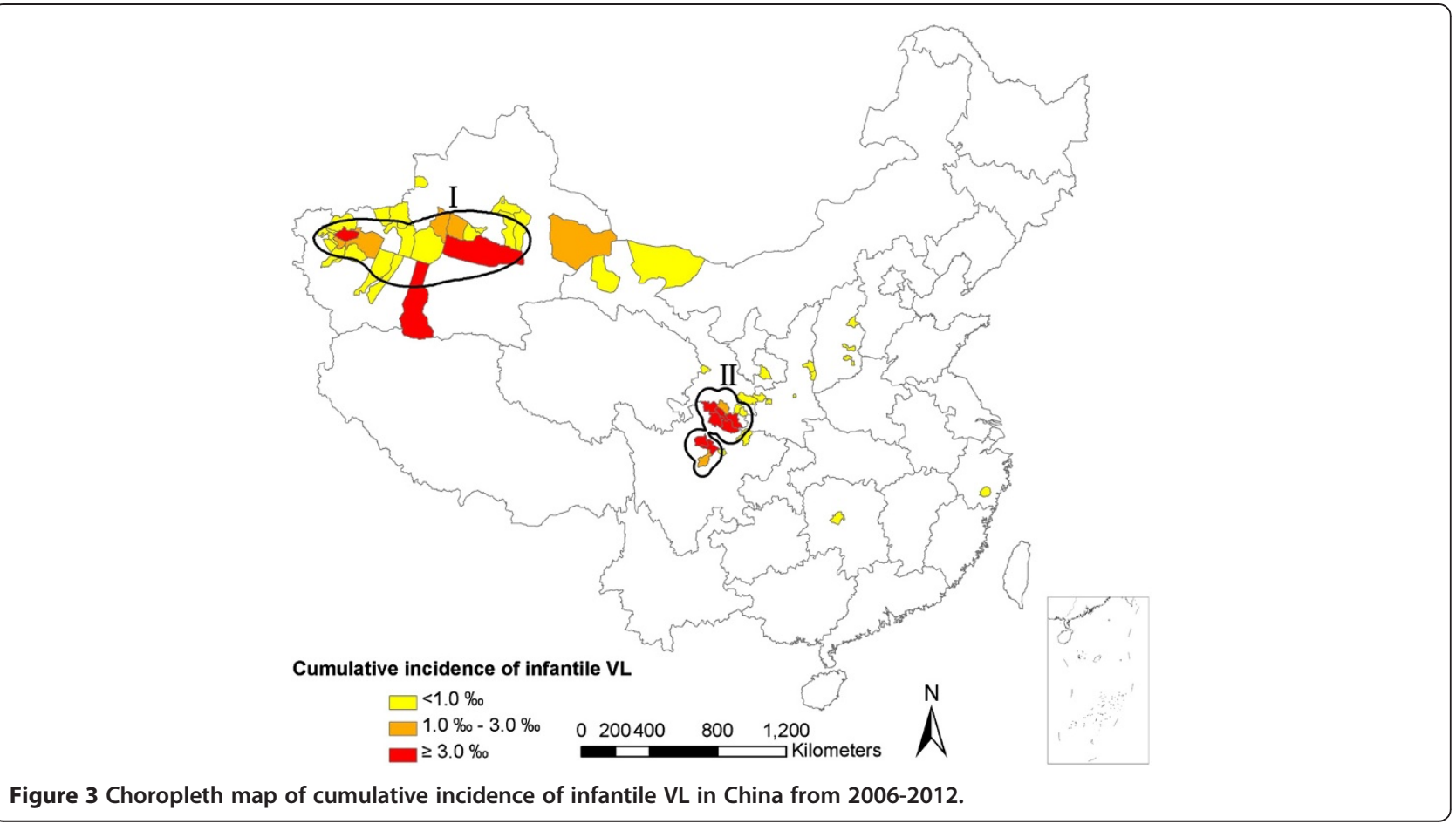

\section{Discussion}

VL causes 500000 cases each year with more than $90 \%$ in India, Bangladesh, Brazil, Nepal and Sudan [4]. At present, a VL elimination program was launched by The WHO South-East Asia Region in 2005 [12,13], which indicates that the global elimination of VL is put on the agenda. However, in China and some other endemic countries, VL has been neglected and seems to have spread in recent years [14-17]. In the early 1950s, there were approximately $530000 \mathrm{VL}$ cases distributed in more than 660 counties of 16 provinces in China [7]. At that time, governments at different levels paid a great deal of attention to VL control to initiate the national control program. VL in China can be classified into three types by different topographic features, Leishmania species, vector species and reservoir hosts [18,19], namely anthroponotic type VL (AVL), mountainous subtype of zoonotic VL (MST-ZVL) and desert sub-type of zoonotic VL (DST-ZVL) [20]. In AVL endemic areas, the control strategy placed emphasis on mass screening and treatment of patients, combined with vector control using indoor or outdoor spraying of insecticides [10]. A large number of patients were found to be infected and were treated using sodium stibogluconate [21], meanwhile the density and natural infection rate of sandflies gradually decreased [22], as a result, prevalence of AVL was effectively controlled by the end of the 1950s in China. On the contrary, because of the exophilic behaviour of the sandfly in MST-ZVL and DST-ZVL endemic areas, there was little effect on vector control by spraying of insecticides [23,24]. In MST-ZVL endemic areas, we adopted the strategy with emphasis on extinction of infected dogs and prohibition of raising domestic dogs, combined with deltamethrin bathing for dogs, which markedly interrupted the transmission of MSTZVL $[25,26]$. Although there were no available means to control DST-ZVL, because of the unknown source of infection and the wild nature of the vector, the number of patients reported was low and they could easily recover through treatment in hospitals. The various control strategies for different types of VL had made great contributions to elimination of VL in China by the end of 1950s, which will be of referential significance to current

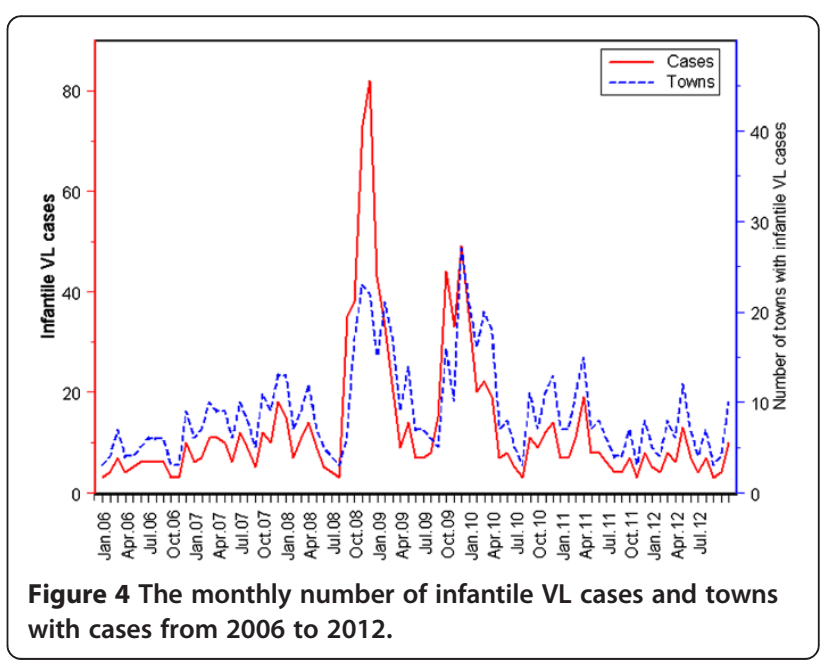


Table 3 Deaths of infantile VL in China from 2006-2012

\begin{tabular}{lcccc}
\hline $\begin{array}{l}\text { Province } \\
\text { (Autonomous Region) }\end{array}$ & County & Gender & Year of death & $\begin{array}{c}\text { Age at } \\
\text { death }\end{array}$ \\
\hline Sichuan & Heishui & female & 2007 & 12 months \\
Xinjiang & Kuerle & female & 2007 & 10 months \\
Xinjiang & Urumqi & male & 2007 & 13 months \\
\hline
\end{tabular}

VL control. Following the mass prevention and control, VL was basically eliminated in China. Since then, because of the decreasing incidence, VL control has been ruled out from the priority agenda. Due to lack of a sound surveillance system and resources for control activities, VL is now one of the neglected tropical diseases in China. According to the data collected from 1990 to 1999 , VL was prevalent or sporadically endemic in 43 counties of 6 western provinces (autonomous regions), which were Xinjiang, Gansu, Sichuan, Shaanxi, Shanxi and Inner Mongolia [10]. More importantly, re-emergence and outbreaks of VL occur intermittently in China [27-29]. In recent years, two outbreaks of VL took place in west Xinjiang Uygur Autonomous Region, namely Shache County of Kashgar Region in 2006 and Jiashi County of Kashgar Region in 2008 to 2009.

It is feasible to evaluate the normative of $\mathrm{VL}$ cases reporting in China, as VL is a notifiable disease by law. VL cases have to be reported by CDC and hospitals at different levels since 2006, so the completeness and reality can be largely ensured. The results of assessment showed a low underreporting rate, a high completeness and concordance rate, so the quality of available data from NIDRS is relatively ideal for analysis on endemic characteristics. Nevertheless, VL is often a hidden health problem, which can unfold without being noticed for many years [30], it should be admitted that the actual VL endemic situation might be greatly underestimated in China, which is similar in other endemic countries [31,32]. Poverty is a major determinant for VL endemic regions, most VL patients and threatened communities live in poverty-stricken regions of west China, with poor access to hygiene services, and with extremely poor levels of diagnosis and treatment, which easily leads to misdiagnosis and missed diagnosis for VL, especially for those too weak e.g. infants, to express their feelings. At the same time, results of a previous pilot study showed that the asymptomatic infection rate of VL was fairly high [33,34]. Therefore, mass screening among high risk groups based on symptom monitoring and clue investigation should be conducted to verify whether the current infantile VL cases in China are the tip of the iceberg. In addition, it is necessary to compulsorily register the identity card number of cases during case reporting in NIDRS, which will be of benefit for excluding repeated cases.

It is crucial to know about the current diagnostic approaches for VL employed in China. At present, definitive diagnosis has relied on identification of the parasite by microscopy in organ smears in China. However, it was too difficult to collect and identify parasites in bone marrow for almost all hospitals and CDC laboratories in endemic areas. Of these 1093 infantile VL cases, only 8 cases were detected by etiological diagnosis. As was shown from records of NIDRS, we could merely get limited information of Leishmania was detected by microscopy. However, it was still unclear which genus they belonged to. Fortunately, rK39 dipstick was one of the most rapid and easy to interpret diagnostic methods, with a relatively high sensitivity and specificity. Since 2000, many field trial results had proved the rK39 dipstick's effect on diagnosis of VL in China, comparing with that of pathogenic examination, the concordance rate ranged from $96.07 \%$ to $100.00 \%$ [35-38]. rK39 dipstick had been extensively employed to detect Leishmania-specific antibodies, and played an irreplaceable role in diagnosis of VL and suspected VL cases in China. In addition, immunological methods of enzyme linked immunosorbent assay (ELISA) were used for detection of specific antibodies of VL cases [39,40]. In recent years, molecular method was also established for the detection of Leishmania species [41-44]. The samples were tested by PCR using specific pairs of primers, for detecting Leishmania-specific DNA. However, the application of molecular techniques was greatly restricted in endemic areas, due to the lack of technicians and equipment.

At present, AVL is endemic in the old oasis areas of west Xinjiang Uygur Autonomous Region, mainly affecting the elder children and adolescents. MST-ZVL is endemic in mountainous areas of southern Gansu Province and northern Sichuan Province, most cases are children less than 10 years old, and the incidence rates for infants are relatively high, ranging from $66.7 \%$ to $85.4 \%$ [45-47]. DSTZVL is endemic in certain desert areas of northwest China, and more than $90 \%$ cases are infants $[48,49]$. According to the endemic types of each county, 56.63\% (619/1093) cases come from DST-ZVL endemic counties, 37.69\% (412/ 1093) from MST-ZVL endemic counties, and only $5.67 \%$ (62/1093) from AVL endemic counties. ZVL is the visibly more serious threat to infants of endemic regions in China, rather than AVL. It is shown that 86.92\% (950/1093) of VL cases are infants under 2 years of age, who can not express discomfort and suffering, except crying, which can easily lead to a weakened immune system, and even to death. From the gender perspective, there is no statistically significant difference over time, male and female infants are at the same degree of being threatened by VL. Accordingly, active surveillance and detection should be strengthened for infants in mountainous areas of southern Gansu Province, northern Sichuan Province and in desert areas of west Xinjiang Uygur Autonomous Region, targeting detection, diagnosis and treatment of infantile cases at the early stage so as to protect the health of infants. In China, 
sodium stibogluconate was recommended and provided as the first-line treatment medicine to patients. Sodium stibogluconate (containing stibonium with $100 \mathrm{mg} / \mathrm{ml}$ ) was uniquely produced by a government-assigned drug factory. It was reported that the treatment effect was fairly good, and more than $97 \%$ patients had been completely cured in two courses of treatment [50,51]. Of course, in the case of relapse, patients will be advised treatment with a secondline medicine, such as amphotericin B [52,53]. It was extensively confirmed that the sodium stibogluconate was relatively safe, and there was no evidence to relate the reported 3 deaths with drug toxicity in this study.

The peak of sandfly density appears from June to September in China [54], which indicates the start of the incidence peak. The temporal clustering and seasonality for infantile VL cases showed most cases occurred in the period of September to February of the next year, which is consistent with results of previous studies [48,49]. The wild Phlebotomus chinensis is the major vector of MSTZVL, and the wild Phlebotomus wui and Phlebotomus alexandri are the vectors of DST-ZVL [55]. Compared with domestic and semi-domestic sandflies of AVL, vectors of ZVL inhabit the wild, with a large quantity and powerful invasiveness [54]. Unfortunately, people live in poor housing, lack individual protection and consciousness and do not use bed nets in endemic areas in China. In particular, infants are usually directly exposed in courtyards without any protection in summer, when they are at the greatest risk of acquiring sandflies bites and infection. Furthermore, spraying insecticide in ZVL endemic areas showed low effectiveness due to the sandfly's breeding nature. In the light of current control status, integrated strategies on the control of vector density, health education and scale-up of insecticide-treated bed nets should be considered and trialed.

At the end of the 20th century there were 43 counties where VL prevailed, cases were in all age groups, and AVL was particularly serious at that time. During the study period, 56 counties reported infantile VL cases, which showed that the geographical range of notified infantile VL has significantly expanded in the last decade in China. As shown from the monthly number of infantile VL cases reported, there is a striking variation over time. In general, the endemic situation is stable, except for two peak onsets in certain months. Meanwhile, we also found a high consistency between the monthly number of cases and towns where cases were reported. In other words, as more monthly infantile VL are cases reported, more endemic towns become apparent. It is suggested that if control efforts are not strengthened, the spatial spread of infantile VL will be inevitable in China. There were two main endemic zones identified for infantile VL in China during the study period, indicating the priority areas for investment and targeting.

\section{Conclusions}

$\mathrm{VL}$ is a neglected disease and affects the poorest of the poor in western China. The endemic situation of infantile VL is serious, with several active foci identified in China. VL has emerged as a severe threat to infants in endemic regions. Control activities and investments are far from adequate for the current demand. Along with poverty, malnutrition and population growth of non-immunized newborns, infantile VL tends to spread. It is particularly urgent to focus much more attention on this situation and take appropriate actions to control VL in key endemic areas of China.

\section{Consent}

VL was a notifiable infectious disease in China. According to the law, when infants were diagnosed as VL, their detailed information will be provided by parents or guardians, for filling in report cards. The premise was that parents or guardians had learnt and agreed with the items about $\mathrm{CDC}$ at different levels were responsible for the collection, analysis and notification of the incidence situation to public. In addition, we did not carry out any field investigation, detection and experiments on patients, it was not necessary to obtain consents from infant's parents or guardians.

\section{Competing interests}

All authors declare that there are no competing interests in this study.

\section{Authors' contributions}

QF designed the study and wrote the first version of the manuscript. QF, YYH and SZ implemented the study. SZL, WPW and LHT reviewed the manuscript. SZ, YF and LPZ collected population data of infants and supervised data entry. All authors have read and approved the final manuscript.

\section{Acknowledgments}

We thank Prof. Xin-Ping Zuo, Wen-Lin Zhang (CDC of Xinjiang Uygur Autonomous Region) and Kaisar (CDC of Kashgar Region), for their help in field work. We thank Prof. Yisilayin Osman for providing precious data and references, Dr. Ying-Jun Qian for refining suggestions. We also thank all of the reviewers who have provided valuable comments for this paper. This work was supported by the National S \& T Major Program (Grant No. 2012ZX10004-220 and 2008ZX10004-011), and the Young Doctor Training Subject of Shanghai Medicine and Health Development Foundation.

\section{Author details}

${ }^{1}$ National Institute of Parasitic Diseases, Chinese Center for Disease Control and Prevention, Shanghai 200025, People's Republic of China. ${ }^{2}$ Key Laboratory of Parasite and Vector Biology, Ministry of Health, Shanghai 200025, People's Republic of China. ${ }^{3}$ WHO Collaborating Center for Malaria, Schistosomiasis and Filariasis, Shanghai 200025, People's Republic of China. ${ }^{4}$ Center for Disease Control and Prevention of Xinjiang Uygur Autonomous Region, Urumqi 830002, People's Republic of China. ${ }^{5}$ Center for Disease Control and Prevention of Gansu Province, Lanzhou 730020, People's Republic of China. ${ }^{6}$ Center for Disease Control and Prevention of Sichuan Province, Chengdu 610041, People's Republic of China.

Received: 22 March 2013 Accepted: 12 May 2013

Published: 17 May 2013 
References

1. Desjeux P: Leishmaniasis. Public health aspects and control. Clin Dermatol 1996, 14(5):417-423.

2. Alvar J, Yactayo S, Bern C: Leishmaniasis and poverty. Trends Parasitol 2006, 22(12):552-557.

3. Boelaert M, Criel B, Leeuwenburg J, Van Damme W, Le Ray D, Van der Stuyft P: Visceral leishmaniasis control: a public health perspective. Trans $R$ SoC Trop Med Hyg 2000, 94(5):465-471.

4. Bern C, Maguire JH, Alvar J: Complexities of assessing the disease burden attributable to leishmaniasis. PLoS Negl Trop Dis 2008, 2(10):e313.

5. World Health Organization: Control of the leishmaniasis. World Health Organ Tech Rep Ser 2010, 949:186.

6. Guerin PJ, Olliaro P, Sundar S, Boelaert M, Croft SL, Desjeux P, Wasunna MK, Bryceson AD: Visceral leishmaniasis: current status of control, diagnosis, and treatment, and a proposed research and development agenda. Lancet Infect Dis 2002, 2(8):494-501.

7. Wang ZJ, Xiong GH, Guan LR: The epidemiology and control achievements of visceral leishmaniasis of People's Republic of China. Chin J Epidemiol 2000, 21(1):51-54.

8. Xu ZB: Present situation of visceral leishmaniasis in China. Parasitol Today 1989, 5(7):224-228

9. Guan LR, Shen WX: Recent advances in visceral leishmaniasis in China. Southeast Asian J Trop Med Public Health 1991, 22(3):291-298.

10. Guan LR, Qu JQ, Chai JJ: Leishmaniasis in China: present status of prevalence and some suggestions on its control. End Dis Bull 2000, 15:49-52.

11. Zhang LM, Leng YJ: Eighty-year research of phlebotomine sandflies (Diptera: Psychodidae) in China (1915-1995). II. Phlebotomine vectors of leishmaniasis in China. Parasite 1997, 4(4):299-306.

12. Picado A, Dash AP, Bhattacharya S, Boelaert M: Vector control interventions for visceral leishmaniasis elimination initiative in South Asia, 2005-2010. Indian J Med Res 2012, 136(1):22-31

13. Mondal D, Singh SP, Kumar N, Joshi A, Sundar S, Das P, Siddhivinayak H, Kroeger A, Boelaert M: Visceral leishmaniasis elimination programme in India, Bangladesh, and Nepal: reshaping the case finding/case management strategy. PLoS Negl Trop Dis 2009, 3(1):e355.

14. Romero GA, Boelaert M: Control of visceral leishmaniasis in latin americaa systematic review. PLOS Negl Trop Dis 2010, 4(1):e584.

15. Dujardin JC, Campino L, Canavate C, Dedet JP, Gradoni L, Soteriadou K, Mazeris A, Ozbel Y, Boelaert M: Spread of vector-borne diseases and neglect of Leishmaniasis, Europe. Emerg Infect Dis 2008, 14(7):1013-1018.

16. Guan LR: Present situation of visceral leishmaniasis and prospect for its control in China. Chin J Parasitol Parasit Dis 2009, 27(5):394-397.

17. Werneck GL: Geographic spread of visceral leishmaniasis in Brazil. Cad Saude Publica 2010, 26(4):644-645

18. Lu HG, Zhong L, Guan LR, Qu JQ, Hu XS, Chai JJ, Xu ZB, Wang CT, Chang KP: Separation of Chinese Leishmania isolates into five genotypes by kinetoplast and chromosomal DNA heterogeneity. AmJTrop Med Hyg 1994, 50(6):763-770.

19. Wang JY, Feng Y, Gao CH, Jin CF, Chen SB, Bao YF: Asymptomatic Leishmania infection in human population of Wenxian County, Gansu Province. Chin J Parasitol Parasit Dis 2007, 25(1):62-64.

20. Wang JY, Cui G, Chen HT, Zhou XN, Gao CH, Yang YT: Current epidemiological profile and features of visceral leishmaniasis in people's republic of China. Parasit Vectors 2012, 5:31

21. Jiang ZP: Historical materials of major infectious diseases of Shandong Province in modern times. Chin J Med Hist 2002, 32(2):119-121.

22. Shao QF, Wang ZL, Han SL, Wang ZL: Experimental effect of killing sandfly using deltamethrin. Chin J Parasitol Parasit Dis 1985, 3(3):228-230.

23. Jin CF, Xiong GH: Effect evaluation of deltamthrin and DDT in the control of periwild sandfly Phlebotomus chinensis. End Dis Bull 1991, 6(2):113-116.

24. Xiong GH, Jin CF: Studies on deltamethrin in the control of peri-wild Phlebotomus chinensis. Chin J Parasitol Parasit Dis 1987, 5(3):176-179.

25. Xiong GH, Jin CF, Hong YM, Su ZW, Xue PZ, Xie WK, Zhang AZ, Li GR, Gao $B$ : Studies on the deltamethrin-medicated bath of domestic dogs for interrupting visceral leishmaniasis transmission. Chin J Parasitol Parasit Dis 1995, 13(3):178-181.

26. Chen SB, Li F, He JP, Chen XW, Wang DG, Wei LS, Yang HP, Guan LR: Experimental study on prevention of dog-sandfly contact by deltamethrin collar. End Dis Bull 2001, 16(3):17-19.

27. Guan LR, Zuo XP, Yimamu: Reemergence of visceral leishmaniasis in Kashi Prefecture, Xinjiang. Chin J Parasitol Parasit Dis 2003, 21(5):285.
28. Wang JY, Gao CH, Yang YT, Chen HT, Zhu XH, Lv S, Chen SB, Tong SX, Steinmann $P$, Ziegelbauer $K$, et al: An outbreak of the desert sub-type of zoonotic visceral leishmaniasis in Jiashi, Xinjiang Uygur Autonomous Region, People's Republic of China. Parasitol Int 2010, 59(3):331-337.

29. Zheng CJ, Wang LY, Xu X, Zhu XH, Wu WP: Visceral leishmaniasis in China during 2004-2007. Chin J Parasitol Parasit Dis 2009, 27(4):344-346.

30. Marlet MV, Wuillaume F, Jacquet D, Quispe KW, Dujardin JC, Boelaert M: A neglected disease of humans: a new focus of visceral leishmaniasis in Bakool, Somalia. Trans R Soc Trop Med Hyg 2003, 97(6):667-671.

31. Mosleh IM, Geith E, Natsheh L, Abdul-Dayem M, Abotteen N: Cutaneous leishmaniasis in the Jordanian side of the Jordan Valley: severe under-reporting and consequences on public health management. Trop Med Int Health 2008, 13(6):855-860.

32. Singh SP, Reddy DC, Rai M, Sundar S: Serious underreporting of visceral leishmaniasis through passive case reporting in Bihar, India. Trop Med Int Health 2006, 11(6):899-905

33. Fu Q, Wu WP, Tong SX, Osman I, Zhang S, Iskender, Kaisar: Investigation on the infection status of visceral leishmaniasis in humen in Kashgar pilot, Xinjiang Uygur Autonomous Region, China. J Pathog Biol 2008, 7(12):758-760.

34. Qu JH, Wang HF, Zhao GH, Zhong WX, Kaisar, Dou HP, Cui Y, Li J, Kong FH: Survey of Leishmania donivani infection in humans in Kashi City of Xinjiang Uygur Autonomous Region. Chin Trop Med 2010, 10(11):1324-1327.

35. Li GR, Duan M, Jiang CD, Li L, Li Y, Zhang LZ, Lan YH, Zhang FN: Recombinant antigen (rK39) dipstick for diagnosis of leishmaniasis. Parasito Infect Dis 2005, 3(1):14-15.

36. Zhang FN, Xiao N, Chen YL: Assessment of results of detection of leishmaniasis pateints with rk39 dipstick. Chin Trop Med 2008, 8(5):710-711.

37. Zuo XP, Yimam S, Kaiser K, Zhang S, Jiao W, Qu JQ, Chang KP, Chai JJ: Application of rK39 dipstick on diagnosis and epidemiological survey of kala-azar in Xinjiang, China. J Trop Dis Parasitol 2007, 5(1):19-22.

38. Qu JQ, Guan LR, Imamu S, Zuo XP, Chai JJ, Chen SB, Shinichiro K, Katakura K, Matsumoto Y, Reed SG, et al: Rapid screening with a recombinant antigen (rK39) for diagnosis of visceral leishmaniasis using dipstick. Chin J Parasitol Parasit Dis 2000, 18(3):155-158.

39. Israyil O, Zhang CW, Kaisar K, Zhang S, Ruzigu Z, Iskender, Wu WP: Investigation in a new kala-azar focus in Shache County of Xinjiang End Dis Bull 2007, 22(5):72-74.

40. Qu JQ, Xu YX, Bao YF, Yang YT, Wang JY: Application of monoclonal antibodies against Leishmania donovani II. Detection of circulating antigen in sera of visceral leishmaniasis before and after treatment. Chin J Parasitol Parasit Dis 1991, 9(1):21-23.

41. Gao CH, Wang JY, Yang YT, Bao YF: Study on PCR method for detecting the asymptomatic Infection of Leishmania infantum. Chin J Parasitol Parasit Dis 2006, 24(2):92-96.

42. Wang JY, Chen SB, Gao CH, Jin CF, Feng Y, Zhang CJ, He HX, Yang CM, Yang YT, Bao YF: Survey on the Leishmania infantum asymptomatic infection in dogs in Wenxian county of Gansu Province. Chin J Zoonoses 2006, 22(8):734-737.

43. Lu DM, Hu XS, Qiao ZD: Analysis of leishmania species and strains from China by RAPD technique. Chin J Parasitol Parasit Dis 2001, 19(5):290-293.

44. Wang JY, Ha Y, Gao CH, Wang Y, Yang YT, Chen HT: The prevalence of canine Leishmania infantum infection in western China detected by PCR and serological tests. Parasit Vectors 2011, 4:69.

45. Wu YX, Zhang AZ, Li GR, Hu XS, Kan B, Hu XM, Jiang NF, Zhang XL: Current situation of kala-azar in Wenchuan County of Sichuan, China. End Dis Bull 1991, 6(4):59-63.

46. Chen SB: Current status of visceral leishmaniasis in Gansu Province. End Dis Bull 1995, 11(2):114-116.

47. Wei LS, Ren WW, Liu PZ: A survey of prevailing status and factors of kala-azar disease made in Longnan Prefecture of Gansu Province,China. End Dis Bull 1993, 8(3):73-74.

48. Chai JJ, Zuo XP, Zhang S, Zhao JP, Zhang JM: The desert type kala-azar in Xinjiang, China. End Dis Bull 1997, 12(4):27-32.

49. Osman Y, Hou YY: Retrospective analysis of prevalence of visceral leishmaniasis in Xinjiang from 2005 to 2010. Bull Dis Control Prev 2011, 26(4):3-6.

50. Wang ZJ, Wu ZJ: Review of investigation and research on Kala-azar of the People's Republic of China. Beijing: Science and Technology Press; 1958:1-2.

51. Wu YX, Chen M: Questions in treatment of children kala-azar. J Practic Parasit Dis 1994, 2(4):40-41. 
52. Liu XL, He SQ: Treatment of kala-azar combining sodium stibogluconate and amphotericin B. End Dis Bull 2007, 22(6):113.

53. Guan LR, Wang JY, Yang YT: Current challenges to leishmaniasis control in China. End Dis Bull 2003, 18(1):46-49.

54. Guan LR, Chai JJ, Zuo XP: Progress of biological study on sandflies in Xinjiang Uygur Autonomous Region, China. End Dis Bull 1999, 14(4):87-91.

55. Guan LR: Current status of kala-azar and vector control in China. Bull World Health Organ 1991, 69(5):595-601.

doi:10.1186/1756-3305-6-143

Cite this article as: Fu et al:: Endemic characteristics of infantile visceral leishmaniasis in the People's Republic of China. Parasites \& Vectors 2013 6:143.

\section{Submit your next manuscript to BioMed Central and take full advantage of:}

- Convenient online submission

- Thorough peer review

- No space constraints or color figure charges

- Immediate publication on acceptance

- Inclusion in PubMed, CAS, Scopus and Google Scholar

- Research which is freely available for redistribution 